\title{
Journal of Ecology \\ COMMON GARDEN EXPERIMENTS TO STUDY LOCAL ADAPTATION NEED TO ACCOUNT FOR POPULATION STRUCTURE
}

\author{
Pierre de Villemereuil ${ }^{1}$, Oscar E. Gaggiotti ${ }^{2}$, and Jérôme Goudet ${ }^{3}$ \\ ${ }^{1}$ Institut de Systématique, Évolution, Biodiversité (ISYEB), École Pratique des Hautes Études / PSL, MNHN, CNRS, \\ Sorbonne Université, Université des Antilles, Paris, France \\ ${ }^{2}$ Scottish Oceans Institute, University of St Andrews, Fife, KY16 8LB, United Kingdom \\ ${ }^{3}$ Department of Ecology and Evolution \& Swiss Institute of Bioinformatics, University of Lausanne, 1015 Switzerland
}

\begin{abstract}
1. Common garden experiments are valuable to study adaptive phenomenon and adaptive potential, in that they allow to study local adaptation without the confounding effect of phenotypic plasticity. The $\mathrm{Q}_{\mathrm{ST}}-\mathrm{F}_{\mathrm{ST}}$ comparison framework, comparing genetic differentiation at the phenotypic and molecular level, is the usual way to test and measure whether local adaptation influences phenotypic divergence between populations.

2. Here, we highlight that the assumptions behind the expected equality $Q_{S T}=F_{S T}$ under neutrality correspond to a very simple model of population genetics. While the equality might, on average, be robust to violation of such assumptions, more complex population structure can generate strong evolutionary noise.
\end{abstract}

Synthesis We highlight recent methodological developments aimed at overcoming this issue and at providing a more general framework to detect local adaptation, using less restrictive assumptions. We invite empiricists to look into these methods and theorists to continue developing even more general methods.

Keywords: Local adaptation, common garden, $\mathrm{Q}_{\mathrm{ST}}-\mathrm{F}_{\mathrm{ST}}$ comparison, population structure, phenotypic divergence, population genetics, quantitative genetics.

\section{Introduction}

19 There are three main possible responses from organisms subjected to climate change (Parmesan 2006; Aitken, Yeaman, Holliday, Wang, and Curtis-McLane 2008): they can (i) disperse and change their range limits, match21 ing the new geographic repartition of their ecological niche, (ii) quickly acclimate to the new climatic condi22 tions through (possibly transgenerational) phenotypic plasticity or (iii) in the longer run, evolve genetically 23 to match the new climatic conditions through an adaptive process. Predicting the likeliness of these three out-

24 comes (or any combination thereof) is challenging and requires both reliable models and detailed information This article has been accepted for publication and undergone full peer review but has not been through the copyediting, typesetting, pagination and proofreading process, which may lead to differences between this version and the Version of Record. Please cite this article as doi: $10.1111 / 1365-2745.13258$

This article is protected by copyright. All rights reserved. 
Fortunately, "common gardens", experimental settings in which offspring from different populations are raised under the same (sets of) environmental conditions, allow to circumvent this difficulty and to assess the amount of adaptive genetic variation that exists among populations (Kawecki and Ebert 2004; Savolainen, Lascoux, and Merila 2013; de Villemereuil, Gaggiotti, Mouterde, and Till-Bottraud 2016). A particular challenge in the analysis of such experiment is to distinguish between neutral and adaptive genetic variation among populations. Indeed, even in absence of selection, and as a result of the combined effect of drift, mutation and migration, populations are expected to diverge from a phenotypic point of view, just as they do from a genetic one (Lande 1992). As the fixation index $\left(\mathrm{F}_{\mathrm{ST}}\right)$ measures the genetic divergence between populations from a molecular point of view, the phenotypic divergence can be measured through a parameter named $\mathrm{Q}_{\mathrm{ST}}$ (Spitze 1993). Both can be defined as the ratio of the between-population genetic variance over the total genetic variance (Lande 1992; Spitze 1993):

$$
\mathrm{Q}_{\mathrm{ST}}=\frac{V_{\mathrm{B}}}{V_{\mathrm{B}}+2 V_{\mathrm{A}}},
$$

${ }_{41}$ where $V_{\mathrm{A}}$ is the within-population additive genetic variance and $V_{\mathrm{B}}$ is the between population genetic variance. ${ }_{42}$ Moreover, in the absence of selection (i.e. for a purely neutral trait), it is expected that $\mathrm{F}_{\mathrm{ST}}=\mathrm{Q}_{\mathrm{ST}}$ (Whitlock 43 2008). Thus, hypothesis testing based on a null hypothesis of neutrality, as first suggested by Spitze (1993) 44 has generated a lot of literature focused on the $\mathrm{Q}_{\mathrm{ST}}-\mathrm{F}_{\mathrm{ST}}$ comparison (reviewed in Leinonen, O'Hara, Cano, 45 and Merilä 2008; Leinonen, McCairns, O’Hara, and Merilä 2013). In these studies, a phenotypic trait with $\mathrm{Q}_{\mathrm{ST}}$ significantly larger than the $\mathrm{F}_{\mathrm{ST}}$ estimated from neutral markers is considered as being under local adaptation, ${ }_{47}$ while a $\mathrm{Q}_{\mathrm{ST}}$ significantly smaller than $\mathrm{F}_{\mathrm{ST}}$ is taken as a sign of balancing selection (Spitze 1993; Leinonen et 48 al. 2013). This framework of $Q_{S T}-F_{S T}$ comparison has been heavily criticised for being subjected to many 49 issues and limitations. For example, $\mathrm{Q}_{\mathrm{ST}}$ is notoriously difficult to estimate and its (often large) uncertainty so should be carefully accounted for in the comparison (O'Hara and Merilä 2005); it can also be influenced by the effect of dominance and inbreeding (Goudet and Büchi 2006; Goudet and Martin 2006; Santure and Wang 2008) and mutation rates might not be comparable between the Quantitative Trait Loci (QTLs) and the neutral markers used to compute $\mathrm{F}_{\mathrm{ST}}$ (Edelaar, Burraco, and Gomez-Mestre 2011; Edelaar and Björklund 2011). Besides offering criticisms, these studies also provide strategies to overcome some of the limitations, and because such a framework is so crucial to the study of local adaptation, it is still very popular in the literature (Leinonen 


\section{${ }_{62}$ The typical population model}

${ }_{63} \mathrm{Q}_{\mathrm{ST}}$ is typically estimated from common garden phenotypic measurements $Y$, in which each individual $i$

${ }_{64}$ belongs to a distinct natural population of origin $p$. The average population effects $a_{p}$ are considered as being ${ }_{65}$ of genetic origin and their variance $V_{\mathrm{B}}$ is assimilated to the between-population genetic variance. While Spitze 66 (1993) used an ANOVA to compute the very first empirical $Q_{S T}$, it is nowadays common (Leinonen et al. 2013) ${ }_{67}$ to use a mixed model to compute $V_{\mathrm{B}}$ and the within-population additive genetic variance $V_{\mathrm{A}}$, using:

$$
Y_{i}=\mu+u_{i}+a_{p(i)}+e_{i}
$$

where $\mu$ is the model intercept, $u_{i}$ is the individual-level genetic random effect, $a_{p(i)}$ is the population-level genetic random effect (of the population $p(i)$ the individual $i$ belongs to) and $e_{i}$ is the residual. The variance associated with the $a_{p}$ effects is $V_{\mathrm{B}}$ and the variance associated with $u_{i}$ is $V_{\mathrm{A}}$. The assumptions about the 71 population genetics model lie in how the structure of the random effects are specified. Generally, in order 72 to estimate the $a_{p}$ effect in the model, one simply uses a "simple" random effect based on the population ID, 3 which results in the assumption that the $a_{p}$ 's are independent and identically distributed. In a more formal way:

$$
a_{p} \sim \mathcal{N}\left(0, \mathrm{I} V_{\mathrm{B}}\right)
$$

where $\mathrm{I}$ is the identity matrix and $\mathcal{N}$ is the normal distribution. This identity matrix is akin to assuming populations are structured according to an island model, where all equally-sized populations receive and send the same number of migrants from a common pool (see Box 1A). Note that this model is also the one generally used to derive $\mathrm{F}_{\mathrm{ST}}$ estimates (Weir and Cockerham 1984, but see Gaggiotti and Foll 2010; Karhunen and Ovaskainen 2012; Weir and Goudet 2017 for other approaches). In practice, this model is quite robust to slight deviations from its assumptions, as shown by decades of experience from population geneticists using $\mathrm{F}_{\mathrm{ST}}$ (Holsinger and Weir 2009).

Theoretically, the equality $\mathrm{F}_{\mathrm{ST}}=\mathrm{Q}_{\mathrm{ST}}$ under neutrality should be valid, on average, for population structures 


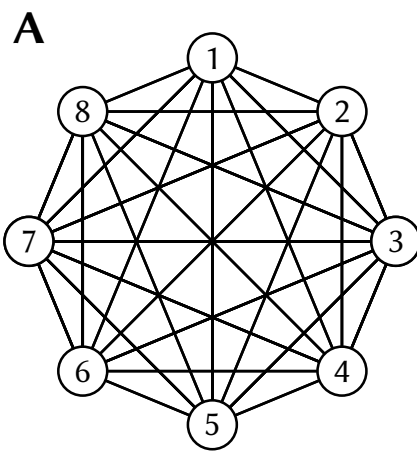

Figure C shows the results. For each scenario (IsIM: Island model, SS1D: SteppingStone), 500 neutral, purely additive and genetically determined traits are simulated with either 10,100 or 1000 (top to bottom row) causal loci and effect sizes drawn from a normal distribution. The red horizontal line on each panel is the genomic $\mathrm{F}_{\mathrm{ST}}$, the violin plots labelled $\mathrm{F}_{\mathrm{ST}}^{Q}$ correspond to $\mathrm{F}_{\mathrm{ST}}$ estimated from causal loci, and the violin plots labelled $\mathrm{Q}_{\mathrm{ST}}$ show the distribution of $\mathrm{Q}_{\mathrm{ST}}$ for each scenario. Dots are the observations. The blue points show the mean value of the observations. For both models, $\mathrm{F}_{\mathrm{ST}}^{Q}$ is essentially unbiased, with more variation when the number of loci encoding the trait is small, as expected. For $\mathrm{Q}_{\mathrm{ST}}$, while more variable that $\mathrm{F}_{\mathrm{ST}}^{Q}$, the estimation for the island model are centered around their expected value, with an empirical $95 \%$ percentile interval $[0.1,0.27]$. For the one-dimensional stepping stone model, despite the mean being close to its expected value, a majority of $\mathrm{Q}_{\mathrm{ST}}$ estimates are smaller than the expected value, with a mode around 0.1 and the range of variation is extremely wide, from 0 to 0.7 , and an empirical $95 \%$ percentile interval $[0.05,0.44]$. Such a variation due to very large evolutionary stochasticity for $\mathrm{Q}_{\mathrm{ST}}$ (note that statistical sampling errors have been minimized here, since $V_{A}$ is the phenotypic variance and $V_{B}$ is estimated from 20 populations, so using the statistical framework in Equation 2 would most likely aggravate the issue) makes carrying a test for the null hypothesis $\mathrm{Q}_{\mathrm{ST}}=\mathrm{F}_{\mathrm{ST}}$ prone to a high level of type I errors, if not accounting for such effect of population structure. Indeed, for the island model, $5 \%$ of the simulated $\mathrm{Q}_{\mathrm{ST}}$ are significant at the $5 \%$ nominal level when using the modified Lewontin-Krakauer test proposed by Whitlock and Guillaume (2009), while 30\% are significant for the one dimensional stepping-stone model. 
88

89 than for $\mathrm{F}_{\mathrm{ST}}$.

\section{Toward a solution: more general strategies}

92 It is difficult to come up with a universal model of neutral evolution, that can account for any sort of population 93 structure. However, there have been some methodological developments that aim at testing local adaptation 94 in a common garden setting with more general assumptions about population structure. Here, we wish to

Ovaskainen, Karhunen, Zheng, Arias, and Merilä (2011) method is not based on a direct $\mathrm{Q}_{\mathrm{ST}}-\mathrm{F}_{\mathrm{ST}}$ compar-

97 ison. Instead, it is based on a theoretically motivated neutral model of phenotypic divergence that allows for

98 differences in migration and drift among populations, as well as preferential migration between populations.

To achieve this, the identity matrix I is replaced by a between-population relatedness matrix (here noted B) 100 in Equation 3:

$$
a_{p} \sim \mathcal{N}\left(0, \mathrm{~B} V_{\mathrm{B}}\right),
$$

101 To estimate this matrix from neutral marker data the same authors propose an extension of the F-model (Gag102 giotti and Foll 2010) that also allows the simultaneous estimation of the other parameters associated with 103 Equation 2. Because $\mathrm{B}$ is a matrix, and not just a single number as is $\mathrm{F}_{\mathrm{ST}}$, this framework offers both a more accurate description of the population structure and more power to detect deviation from neutrality. It does so by alleviating the issues affecting the direct comparison of $\mathrm{Q}_{\mathrm{ST}}$ with $\mathrm{F}_{\mathrm{ST}}$ and provides a statistical test (coined " $S$-test") measuring the deviation of the population means themselves from the neutral expectation. A more recent implementation of the method in R (driftsel, Karhunen, Merilä, Leinonen, Cano, and Ovaskainen 2013) first estimates the matrix using neutral markers and an admixture F-model (Karhunen and Ovaskainen 2012), and then incorporates quantitative trait data to estimate all remaining parameters and further refine the estimate of the matrix. Finally, a new alternative method to estimate the B matrix (which can then be used by driftsel) is provided by the unified approach to characterise population structure and individual relatedness and inbreeding recently put forward by Weir and Goudet (2017). Using this model of neutral evolution ap- 


\section{Conclusion}

131 Since it was first proposed by Spitze (1993), the $Q_{S T}-F_{S T}$ comparison framework has been an invaluable tool 132 to investigate the prevalence and characteristics of local adaptation. Nonetheless, we encourage empiricists to consider the alternative strategies discussed here to better account for various effects of population structure. 134 As these new strategies themselves have their own limitations, we also urge theorists to continue developing 135 new methods to study the phenotypic impact of local adaptation in common garden, while accounting for 136 population structure and evolution stochasticity as accurately as possible.

\section{Authors contribution}

138 PdV led the writing of the manuscript with critical contributions from OEG and JG. JG performed the simula-

139 tion analysis, with analysis from all authors. All authors agreed to the publication. 


\section{Data Availability}

We provide the code for replicating the analysis in Supplementary Information.

\section{References}

Aitken, S. N., Yeaman, S., Holliday, J. A., Wang, T., and Curtis-McLane, S. (2008). Adaptation, migration or extirpation: climate change outcomes for tree populations. Evolutionary Applications 1.1, 95-111.

de Villemereuil, P., Gaggiotti, O. E., Mouterde, M., and Till-Bottraud, I. (2016). Common garden experiments in the genomic era: new perspectives and opportunities. Heredity 116.3, 249-254.

de Villemereuil, P., Mouterde, M., Gaggiotti, O. E., and Till-Bottraud, I. (2018). Patterns of phenotypic plasticity and local adaptation in the wide elevation range of the alpine plant Arabis alpina. Journal of Ecology 106.5, 1952-1971.

Edelaar, P. and Björklund, M. (2011). If FST does not measure neutral genetic differentiation, then comparing it with QST is misleading. Or is it? Molecular Ecology 20.9, 1805-1812.

Edelaar, P., Burraco, P., and Gomez-Mestre, I. (2011). Comparisons between QST and FST-how wrong have we been? Molecular Ecology 20.23, 4830-4839.

Gaggiotti, O. E. and Foll, M. (2010). Quantifying population structure using the F-model. Molecular Ecology Resources $10.5,821-830$.

Goudet, J. and Martin, G. (2006). Under neutrality, QST <= FST when there is dominance in an island model. Genetics $176.2,1371-1374$.

Goudet, J. and Büchi, L. (2006). The effects of dominance, regular inbreeding and sampling design on QST, an estimator of population differentiation for quantitative traits. Genetics 172.2, 1337-1347.

Holsinger, K. E. and Weir, B. S. (2009). Genetics in geographically structured populations: defining, estimating and interpreting F ST. Nature Reviews Genetics 10.9, 639-650.

Hudson, R. R. (2002). Generating samples under a Wright-Fisher neutral model of genetic variation. Bioinformatics 18.2, 337-338.

Josephs, E. B., Berg, J. J., Ross-Ibarra, J., and Coop, G. (2019). Detecting adaptive differentiation in structured populations with genomic data and common gardens. Genetics 211.3, 989-1004.

Karhunen, M., Merilä, J., Leinonen, T., Cano, J. M., and Ovaskainen, O. (2013). DRIFTSEL: an R package for detecting signals of natural selection in quantitative traits. Molecular Ecology Resources 13.4, 746-754.

Karhunen, M., Ovaskainen, O., Herczeg, G., and Merilä, J. (2014). Bringing habitat information into statistical tests of local adaptation in quantitative traits: a case study of nine-spined sticklebacks. Evolution 68.2, 559-568.

Karhunen, M. and Ovaskainen, O. (2012). Estimating population-level coancestry coefficients by an admixture F model. Genetics 192.2, 609-617.

Kawecki, T. J. and Ebert, D. (2004). Conceptual issues in local adaptation. Ecology Letters 7.12, 1225-1241.

Lande, R. (1992). Neutral theory of quantitative genetic variance in an island model with local extinction and colonization. Evolution 46.2, 381-389. 
Leinonen, T., O’Hara, R. B., Cano, J., and Merilä, J. (2008). Comparative studies of quantitative trait and neutral marker divergence: a meta-analysis. fournal of Evolutionary Biology 21.1, 1-17.

Leinonen, T., McCairns, R. J. S., O’Hara, R. B., and Merilä, J. (2013). QST-FST comparisons: evolutionary and ecological insights from genomic heterogeneity. Nature Reviews Genetics 14.3, 179-190.

Martins, H., Caye, K., Luu, K., Blum, M. G. B., and François, O. (2016). Identifying outlier loci in admixed and in continuous populations using ancestral population differentiation statistics. Molecular Ecology 25.20, 5029-5042.

O'Hara, R. B. and Merilä, J. (2005). Bias and precision in QST estimates: problems and some solutions. Genetics 171.3, $1331-1339$.

Ovaskainen, O., Karhunen, M., Zheng, C., Arias, J. M. C., and Merilä, J. (2011). A new method to uncover signatures of divergent and stabilizing selection in quantitative traits. Genetics 189.2, 621-632.

Parmesan, C. (2006). Ecological and evolutionary responses to recent climate change. Annual Review of Ecology, Evolution, and Systematics 37, 637-669.

Santure, A. W. and Wang, J. (2008). The joint effects of selection and dominance on the QST - FST contrast. Genetics 181.1, 259-276.

Savolainen, O., Lascoux, M., and Merila, J. (2013). Ecological genomics of local adaptation. Nature Reviews Genetics 14.11, 807-820.

Spitze, K. (1993). Population structure in Daphnia obtusa: quantitative genetic and allozymic variation. Genetics 135.2, 367-374.

Waples, R. S. and Gaggiotti, O. (2006). What is a population? An empirical evaluation of some genetic methods for identifying the number of gene pools and their degree of connectivity. Molecular Ecology 15.6, 1419-1439.

Weir, B. S. and Cockerham, C. C. (1984). Estimating F-statistics for the analysis of population structure. Evolution 38.6, $1358-1370$

Weir, B. S. and Goudet, J. (2017). A unified characterization of population structure and relatedness. Genetics 206.4, 20852103.

Whitlock, M. C. (1999). Neutral additive genetic variance in a metapopulation. Genetics Research 74.3, 215-221.

- (2008). Evolutionary inference from $Q_{\text {ST. }}$ Molecular Ecology 17.8, 1885-1896.

Whitlock, M. C. and Guillaume, F. (2009). Testing for spatially divergent selection: comparing $Q_{S T}$ to $F_{S T}$. Genetics 183.3, 1055-1063. 